\title{
La boccia como deporte adaptado y sensibilizador en Educación Física en Educación Secundaria
}

\section{Boccia as an adapted and sensitizing sport in Physical Education in Secondary Education}

\author{
Jorge Abellán*, Nieves María Sáez-Gallego y Sebastián Carrión Olivares
}

Universidad de Castilla-La Mancha

\begin{abstract}
Resumen: Los deportes adaptados están siendo incluidos en las programaciones de Educación Física. El presente trabajo evalúa el efecto en las actitudes hacia la discapacidad en Educación Física de la realización de una unidad didáctica de boccia, siguiendo la enseñanza comprensiva de los deportes, y de un torneo inclusivo junto a personas con discapacidad intelectual en el que participó un grupo de estudiantes de educación secundaria. Adicionalmente, se ha recogido su opinión sobre la boccia como contenido deportivo en Educación Física. Los resultados indican que los participantes mejoraron sus actitudes hacia la discapacidad en Educación Física. Además, los participantes indicaron que la boccia contribuye a la educación en valores e igualdad de oportunidades, pero no al desarrollo de habilidades y destrezas básicas. Los estudiantes solo perciben a la boccia como un deporte sensibilizador, por lo que se debe seguir investigando en esta línea para mejorar su opinión como contenido deportivo en Educación Física.

Palabras clave: Deporte paralímpico; inclusión; educación en valores.
\end{abstract}

Abstract: Adapted sports are being included in physical education programming. The present work evaluates the effect in the attitudes toward disabilityin Physical Education of the realization of a didactic unit of boccia following the teaching games for understanding' perspective and an inclusive tournament with people with intellectual impairments of a group of secondary school students. Additionally, student's opinion about boccia has been collected as sports content in Physical Education. The results indicate that participants improved their attitudes toward disability in Physical Education. In addition, participants indicated that the boccia contributes to values education and equal opportunities, but not to the development of basic skills and abilities. The students only perceive the boccia as a sensitizing sport, for which reason it is necessary to continue investigating in this line to improve their opinion as sports content in Physical Education. Keywords: Paralympic sport; inclusion; education in values.

\section{Introducción}

En este trabajo se presenta una perspectiva novedosa, combinando por primera vez la medición de las actitudes hacia la discapacidad con la opinión de los participantes respecto al deporte adaptado como contenido de Educación Física (en adelante EF). En primer lugar, el estudio de las actitudes hacia la discapacidad en EF en la Educación Secundaria nos ha indicado que las mejores actitudes las han mostrado las alumnas,el alumnado con familiares y/o amigos con discapacidad, yel alumnado de mayor edad (Abellán, Sáez-Gallego y Reina, 2018b). Igualmente, los estudiantes con participación en actividades físico-deportivas inclusivas presentan mejores actitudes hacia la discapacidad en EF, como indican los trabajos previos de Abellán et al. (2018b) y Pérez-Tejero, OrtegaVila y Coterón (2012).

Por otro lado, la aplicación de Unidades Didácticas de deporte adaptado había conseguido éxito a la hora de modificar las actitudes hacia la discapacidad de estudiantes de Educación Secundaria en EF. Por ejemplo, los trabajos utilizando la boccia (González y Baños, 2012) o el fútbol sala para perso-

Dirección para correspondencia [Correspodence address]: Jorge Abellán. Universidad de Castilla-La Mancha (Spain).

E-mail: jorge.abellan@uclm.es nas con ceguera o deficiencia visual (Reina, López, Jiménez, García-Calvo, y Hutzler, 2011) habían mejorado las actitudes hacia la discapacidad de los estudiantes participantes. Incluso experiencias de un solo día de práctica de deportes paralímpicos han logrado modificar positivamente las actitudes hacia la discapacidad de los estudiantes participantes (McKay, Block y Park, 2015). Finalmente, se han ido incorporando actividades de deporte inclusivo en las diferentes programaciones docentes, en las que los estudiantes han compartido práctica deportiva con personas con discapacidad, lo que también ha mejorado sensiblemente las actitudes hacia la discapacidad en EF. Por ejemplo, Santana y Garoz (2013) incluyeron la práctica conjunta de goalball de personas con discapacidad visual y alumnos de Educación Secundaria, mejorando las actitudes hacia la discapacidad de estos últimos.

Recientemente se ha sugerido que los deportes adaptados deberían convertirse en un contenido habitual de las clases de EF. Abellán y Sáez-Gallego (2017) sugieren que los deportes adaptados pueden cumplir una triple función dentro de las clases de EF: pueden actuar como deportes alternativos debido a su novedad en los programas escolares; pueden ser considerados como deportes para trabajar la iniciación deportiva; y finalmente, como ya se ha co- 
mentado, pueden actuar para sensibilizar a los alumnos hacia la discapacidad.

En esta línea, Robles-Rodríguez, Abad-Robles, Giménez y Benito-Peinado (2017) analizaron la percepción de un grupo de estudiantes de Bachillerato sobre los deportes adaptados en comparación con los deportes tradicionales. Tras realizar seis Unidades Didácticas, tres de deportes tradicionales (baloncesto, balonmano y voleibol) y otras tres de deportes adaptados (voleibol sentado, bádminton sentado y goalball) los participantes percibieron que los deportes adaptados fomentaban en mayor medida la igualdad de oportunidades, el respeto y la sensibilización social.

Este trabajo presenta un doble objetivo: por un lado, pretende examinar el efecto de un programa de cambio de actitudes hacia la discapacidad en alumnos de Secundaria a través de la realización de una Unidad Didáctica de boccia, siguiendo la propuesta de Abellán y Sáez-Gallego (2017) para su trabajo a partir de la enseńanza comprensiva de los deportes, e incluyendo un contacto con personas con discapacidad intelectual al participaren un torneo inclusivo de este deporte y, por otro lado, conocer la opinión de los alumnos sobre la utilización del deporte adaptado de la boccia como contribución a la educación en valores y la mejora de las habilidades motrices.

\section{Método}

\section{Participantes}

Veintiocho estudiantes de Educación Secundaria Obligatoria (ESO) de un centro de Castilla-La Mancha (Medad=14,68 \pm $1,219)$ tomaron parte en el estudio. El grupo se componía de 12 chicas $($ Medad $=14,25 \pm 1,138)$ y 16 chicos $($ Medad $=15 \pm$ 1,211). Los participantes se distribuían equitativamente entre aquellos que estaban cursando $2^{\circ}$ de ESO $(N=14$, Medad $=$ $13,71 \pm, 611)$ y aquellos que estaban cursando $3^{\circ}$ de ESO $(N=14$, Medad=15,64 \pm ,842).La selección de la muestra ha seguido un modelo no probabilístico de conveniencia. El $39,3 \%$ de los participantes manifestó tener un familiar con discapacidad, aunque ninguno de ellos afirmó haber tenido nunca un compañero con discapacidad en sus clases de EF, ni en el resto de sus clases, ni haber participado nunca en experiencias de deporte adaptado.

Todos los estudiantes participaron en el estudio voluntariamente y aportaron la autorización firmada por su padre, madre o tutor legal antes de comenzar. Se obtuvieron además los permisos pertinentes del equipo de dirección del centro donde se llevó a cabo la recolección de datos. La presente investigación respeta los acuerdos de la Declaración de Helsinki.

\section{Instrumentos}

Para responder a los objetivos del trabajo se utilizaron tres cuestionarios, dos de ellos para conocer las actitudes hacia la discapacidad de los participantes (Ińiguez-Santiago, Férriz, Martínez-Galindo, Cebrián-Sánchez y Reina, 2017; Reina, Hutzler, Ińiguez-Santiago y Moreno-Murcia, 2016) y uno para conocer su opinión sobre la utilización de los deportes adaptados en las clases de EF (Robles-Rodríguez et al., 2017).

Para medir las actitudes hacia la discapacidad se utilizaron dos cuestionarios. El primero de ellos hace referencia a la versión en español del cuestionario "The Attitudes towards Inclusion of Students with Disabilities in Physical Education" (AISDPE) (Reina et al., 2016). Este cuestionario de actitudes hacia la inclusión de estudiantes con discapacidad en EFa se basa en una escala de 17 ítems que incluye dos componentes: el componente cognitivo (ítems 1, 3, 4, 6, 13, 14 y 15) y el componente conductual (ítems 2, 5, 7, 8, 9, 10, 11, 12, 16 y 17). El segundo instrumento es la "Escala de Actitud hacia el Alumnado con Discapacidad en Educación Física” (EAADEF) (Íńiguez-Santiago et al., 2017). Esta escala incluye cuatro ítems, todos ellos relacionados con el componente conductual.

Para la administración de ambos cuestionarios se empleó una escala Likert de 1 (totalmente en desacuerdo) a 5 (totalmente de acuerdo) y la sentencia previa "En las siguientes situaciones con personas con discapacidad...”. Ambos cuestionarios están enunciados de manera que una mayor puntuación en el mismo significa una actitud menos favorable hacia la discapacidad. En ambos cuestionarios se utilizó la media de las escalas como valor estadístico.

Para medir la opinión de los alumnos respeto a la utilización de la boccia como deporte en EF, se ha utilizado el cuestionario creado y validado por Robles-Rodríguez et al. (2017). Dicho cuestionario está compuesto por 5 dimensiones: Valores (e.g. "la práctica de estos deportes favorece el respeto"), Participación (e.g. "independientemente de mi nivel de juego respecto al de mi compañero suelo participar en las jugadas"), Sensibilización social (e.g. "la práctica de estos deportes facilita la integración de cualquier persona en el grupo"), Reflexión crítica (e.g. "la práctica de estos deportes me ha hecho reflexionar sobre la igualdad de oportunidades de las personas") y Habilidades y destrezas motrices (e.g. "me resulta fácil jugar a estos deportes porque los gestos deportivos son fáciles de realizar"). Cada una de las dimensiones se compone de 5 ítems. Para la administración de este cuestionario se empleó una escala Likert de 1 (totalmente en desacuerdo) a 4 (totalmente de acuerdo). Los ítems con una redacción negativa (e.g. "estos deportes no deberían practicarse en EF") se han recodificado, de manera que una puntuación cercana a 4 siempre expresará una opinión positiva hacia la boccia en EF. 


\section{Procedimiento}

El tiempo empleado para responder a los cuestionarios fue de 30 minutos aproximadamente en cada momento de medida (pre-test y post-test). Las recogidas de datos se realizaron en su clase habitual, en horario lectivo, asegurando las condiciones de aplicación en un lugar tranquilo.

Tras finalizar la toma de datos inicial, el grupo desarrolló una Unidad Didáctica de boccia de siete sesiones siguiendo las directrices de Abellán y Sáez-Gallego (2017), adaptando los contenidos de trabajo al currículo de Secundaria en Castilla-La Mancha y utilizando la enseñanza comprensiva de los deportes como metodología, de acuerdo con Griffin, Mitchell y Oslin (1997). El final de la intervención correspondió con la participación de los estudiantes en un torneo de boccia inclusivo, en el que los alumnos formaron equipos de tres junto a personas con discapacidad intelectual adultas (ratio 2 alumnos/1 persona con discapacidad), compartiendo partidos durante dos horas.

El grupo completó los cuestionarios sobre actitudes hacia la discapacidad (Iñiguez-Santiago et al., 2017; Reina et al., 2016) antes y después de la realización de la Unidad Didáctica de boccia. Finalmente, completó el cuestionario sobre la opinión de los alumnos respecto a la utilización de los paradeportes como contenido de EF (Robles-Rodríguez et al., 2017) después de la Unidad Didáctica.

La administración de los cuestionarios se realizó durante el primer trimestre del curso. El tiempo total de la intervención, entre la medida pre-test, la intervención y el post-test, se realizó en dos meses.

\section{Análisis estadístico}

Para la estadística inferencial en el análisis de las actitudes se ha utilizado la prueba con rangos de Wilcoxon, tras comprobar que la distribución de los datos no era normal para todas las variables con la prueba Shapiro-Wilks. Se ha tomado 0,05 como nivel de significación de Alfa.

Para comparar las diferencias en las puntuaciones otorgadas a cada factor por el total de los participantes en cuanto a la opinión de los participantes sobre los deportes adaptados en EF, se aplicó el test de Friedman, utilizando la prueba de los rangos con signo de Wilcoxon como Post-hoc.

\section{Resultados}

\section{Actitudes hacia la discapacidad}

La Tabla 1 muestra los estadísticos descriptivos (media \pm desviación típica) de la puntuación obtenida en cada una de las dimensiones (AISDPE Cognitiva, AISDPE Conductual y EAADEF) antes y después de la intervención. Los resultados indican que el grupo experimenta una mejora significativa en las actitudes hacia la discapacidad en la dimensión AISDPE cognitiva, además de un ligero aumento de la puntuación en la dimensión AISDPE conductual, aunque sin diferencias significativas, mientras que la escala EEADEF empeora sus valores sutilmente, también sin diferencias significativas entre los momentos de medida.

Tabla 1. Estadística descriptiva e inferencial de las actitudes hacia la discapacidad, antes y después de la intervención.

\begin{tabular}{lcccc}
\hline & \multicolumn{2}{c}{ TEST } & \multicolumn{2}{c}{ Wilcoxon } \\
\cline { 2 - 5 } & Pre-test & Post-test & Z & p \\
\hline AISDPE Cognitivo* & $2,267 \pm 0,67$ & $2,057 \pm 0,59$ & $-2,15$ &, 031 \\
AISDPE Conductual & $2,076 \pm 0,69$ & $1,873 \pm 0,63$ & $-1,36$ &, 172 \\
EEADEF & $1,366 \pm 0,58$ & $1,420 \pm 0,76$ &,- 60 &, 546 \\
\hline Nota. Asti
\end{tabular}

Nota. Asterisco $\left(^{*}\right)$ en la variable indica diferencias significativas para $p<, 05$.

Opiniones hacia la boccia como contenido de EF

En la Tabla 2 se muestran la estadística descriptiva e inferencial del total de las puntuaciones obtenidas en los diferentes factores.

Tabla 2. Estadísticos descriptivos $(\mathrm{M} \pm \mathrm{DT})$ de la puntuación obtenida en cada factor para el total de la muestra y valores obtenidos en el Test de Friedman.

\begin{tabular}{lccccc}
\hline & $\mathrm{M} \pm \mathrm{DT}$ & $\begin{array}{c}\text { Rango } \\
\text { Promedio }\end{array}$ & $\mathrm{X}^{2}$ & $\mathrm{gl}$ & $\mathrm{p}$ \\
\hline Valores & $3,68 \pm 0,55$ & 4,04 & & & \\
Participación & $3,59 \pm 0,38$ & 3,50 & & & \\
$\begin{array}{l}\text { Sensibilización } \\
\text { social }\end{array}$ & $3,57 \pm 0,42$ & 3,63 & 39,39 & 4 & $<, 001$ \\
$\begin{array}{l}\text { Reflexión crítica } \\
\begin{array}{l}\text { Habilidades y } \\
\text { destrezas motrices }\end{array}\end{array}$ & $3,44 \pm 0,48$ & 2,86 & & & \\
\hline
\end{tabular}

El test de Friedman muestra diferencias significativas entre los factores. El análisis post-hoc confirma estas diferencias significativas. El análisis post-hoc, realizado por medio de la prueba de los rangos con signo de Wilcoxon, revela diversas diferencias significativas entre los factores analizados. El factor Habilidades y destrezas motrices obtiene peores puntuaciones que el resto de los factores analizados [Valores $(Z=$ $-3,720, p<0,001)$, Participación $(Z=-4,155, p<0,001)$, Sensibilización social $(Z=-3,626, p<0,001)$ y Reflexión crítica $(Z=$ $-3,135, p=0,002)]$. Además, el factor Reflexión crítica obtiene puntuaciones más bajas al compararlo con el factor Valores $(Z=-2,831, p=0,005)$. 


\section{Discusión}

El objetivo del presente trabajo fue analizar el efecto de la realización de una Unidad Didáctica de boccia, acompañada por la participación en un torneo inclusivo junto a personas con discapacidad intelectual, en las actitudes hacia la discapacidad y en la opinión acerca de los deportes adaptados en EF de un grupo de estudiantes de ESO.

Esta investigación presenta una perspectiva novedosa por varios motivos; en primer lugar, es la primera experiencia práctica que utiliza, adaptada a la ESO, la propuesta metodológica que sugiere utilizar la boccia en EF junto a la enseńanza comprensiva de los deportes (de Abellán y Sáez-Gallego, 2017); en segundo lugar, es la primera en la que se combina el estudio de las actitudes hacia la discapacidad en EF junto a la opinión de los estudiantes de los deportes adaptados como contenido de EF en Secundaria, que había sido previamente investigada por separado por Robles-Rodriguez et al. (2017); y en tercer lugar, utiliza el contacto junto a personas con discapacidad intelectual, que de acuerdo con Scior (2011), constituye un tipo de discapacidad que no ha gozado de las experiencias suficientes en investigación de cara a mejorar las actitudes hacia este colectivo (para una excepción, ver Abellán, Sáez-Gallego y Reina, 2018a). Además, combinada dichas novedades con el estudio clásico de los programas de cambio de actitudes hacia la discapacidad en EF, que han conseguido efectos beneficiosos en la mayoría de los casos (Felipe y Garoz, 2014).

Los resultados indican que la intervención realizada ha mejorado las actitudes hacia la discapacidad en EF de los participantes a nivel del factor AISDPE cognitivo. Estos datos están de acuerdo con lo obtenido recientemente por Abellán et al. (2018a), quienes, utilizando los mismos instrumentos utilizados en este trabajo, encontraron que el grupo de estudiantes de ESO que realizó una visita a un centro especial de empleo y participó en una jornada de deporte inclusivo junto a personas con discapacidad intelectual mejoró sus actitudes hacia la discapacidad en EF, también en el factor AISDPE cognitivo. La boccia ya había sido utilizada como deporte en EF y se había conseguido mejorar las actitudes hacia la discapacidad en EF, utilizando también el contacto junto a personas con discapacidad (Santana y Garoz, 2013). Además, se había demostrado que los programas de cambio de actitudes hacia la discapacidad en EF que utilizaban el contacto directo junto a personas con discapacidad, la información sobre ellas y la simulación de la discapacidad por medio del deporte adaptado, conseguían mejorar las actitudes de los participantes (Felipe, Garoz y Tejero-González, 2018). Sin embargo, los resultados del resto de factores analizados en este trabajo hacen que se deba seguir investigando en esta línea. Especialmente contradictorios son los resultados obtenidos a partir de
EAADEF, que empeora levemente sus resultados, aunque no significativamente, tras la realización de la Unidad Didáctica, mientras que el resto de los factores mejoran. Por ello, los resultados deben ser tomados con cautela y se recomienda seguir investigando en esta línea para obtener resultados más concluyentes.

Los resultados en cuanto a las opiniones muestran que los participantes valoran positivamente el deporte de la boccia como contenido de EF relacionado con la educación en valores, ya que los factores con una puntuación más alta son Valores, Participación y Sensibilización social, mientras que los factores Reflexión crítica y, especialmente, Habilidades y destrezas motrices son significativamente peores tras la realización de la intervención. Es probable que la utilización de la enseñanza comprensiva de los deportes, en la que se enseña el deporte priorizando la táctica sobre la técnica tenga influencia en la opinión que muestran los alumnos sobre la contribución de la boccia para la mejora de sus habilidades y destrezas motrices.

Estos resultados están de acuerdo con el análisis realizado por Robles-Rodriguez et al. (2017) quienes también mostraron valores altos en los factores relacionados con la Sensibilización social, Participación y Valores al comparar Unidades Didácticas de deportes adaptados y tradicionales. Tanto en el presente trabajo como en el desarrollado por Robles-Rodríguez et al. (2017) aparece como el factor menos valorado el de Habilidades y destrezas motrices. Aunque Abellán y SáezGallego (2017) indicaron que los deportes adaptados podrían utilizarse desde la triple perspectiva de sensibilizadores, alternativos y de iniciación deportiva, parece que los alumnos consideran que el trabajo de estos deportes se centra en el apartado sensibilizador y de educación en valores, por lo que habría que seguir trabajando en esta línea para conseguir una inclusión con garantías de los deportes como contenido propio de iniciación deportiva dentro de la EF escolar.

\section{Conclusiones}

Teniendo en cuenta la naturaleza exploratoria del trabajo, lo reducido de la muestra y los objetivos propuestos, se puede concluir, que, en esta población de estudio, la realización de una Unidad Didáctica de boccia ha conseguido modificar sus actitudes hacia la discapacidad en EF (a nivel de AISDPE Cognitivo). Además, los participantes han mostrado que identifican la práctica de deportes adaptados como la boccia como una herramienta para la educación en valores, el fomento de la participación y la sensibilización social a través del deporte. Sin embargo, los participantes no valoran tan positivamente su contribución a la reflexión crítica y, especialmente, al desarrollo de las habilidades y destrezas motrices. 


\section{Referencias bibliográficas}

1. Abellán, J. y Sáez-Gallego, N.M. (2017). Los para-deportes como contenido de educación física en primaria: el ejemplo de la Boccia. Didacticae, 2, 134-142. doi:10.1344/did.2017.2.134-142

2. Abellán, J., Sáez-Gallego, N.M. y Reina, R. (2018a). Explorando el efecto del contacto y el deporte inclusivo en Educación Física en las actitudes hacia la discapacidad intelectual en los estudiantes de secundaria. RICYDE. Revista internacional de ciencias del deporte, 53, 233-242.doi: 10.5232/ricyde2018.05304

3. Abellán, J., Sáez-Gallego, N.M. y Reina, R. (2018b). Evaluación de las actitudes hacia la discapacidad en educación física: Efecto diferencial del sexo, contacto previo y la percepción de habilidad y competencia. Cuadernos de Psicología del Deporte, 18(1), 133-140.

4. Felipe, C. y Garoz, I. (2014). Actividad físico-deportiva en programas de cambio de actitudes hacia la discapacidad en edad escolar: una revisión de la literatura. Cultura, Ciencia y Deporte, 9(27), 199-210.doi: 10.12800/ccd.v9i27.462

5. Griffin, L. L., Mitchell, S.A. y Oslin, J. L. (1997). Teaching sport concepts and skills: A tactical games approach. Champaign, IL: Human Kinetics.

6. González, J. y Baños, L. M. (2012). Estudio sobre el cambio de actitudes hacia la discapacidad en clases de actividad física. Cuadernos de Psicología del Deporte, 12(2), 101-108. doi: 10.4321/S157884232012000200011

7. Íniguez-Santiago, M. C., Férriz, R., Martínez-Galindo, M. C., Cebrián-Sánchez, M. y Reina, R. (2017). Análisis factorial de la escala de actitudes hacia el alumnado con discapacidad en educación física (EA-
ADEF). Psychology, Society, \& Education, 9 (3), 493-504.doi: 10.25115/ psye.v9i3.652

8. McKay, C., Block, M. y Parck, J.Y. (2015). The impact of paralympic school day on student attitudes toward inclusion in physical education. Adapted Physical Activity Quarterly,32(4), 331-348. doi: 10.1123/ APAQ.2015-0045

9. Pérez-Tejero, J., Ortega-Vila, G. y Coterón, J. (2012). Diseño y aplicación de un programa de intervención de práctica deportiva inclusiva y su efecto sobre la actitud hacia la discapacidad: El Campus Inclusivo de Baloncesto. Revista Internacional de Ciencias del Deporte, 8 (29),258271.doi. 10.5232/ricyde2012.02905

10. Reina, R., Hutzler, Y., Ińiguez-Santiago, M. C. y Moreno-Murcia, J. A. (2016). Attitudes towards inclusion of students with disabilities in Physical Education Questionnaire (AISDPE): A two-component scale in Spanish. European Journal of Human Movement, 36, 75-87.

11. Reina, R., López, V., Jiménez, M., García, T. y Hutzler, Y. (2011) Effects of awarenessinterventions on children's attitudes toward peers with a visual impairment. International Journal of Rehabilitation Research, 34(3), 235-242.doi:10.1097/MRR.0b013e3283487f49

12. Santana, P. y Garoz, I. (2013). Actitudes hacia la discapacidad e intervención docente desde el deporte adaptado. Revista Internacional de Medicina y Ciencias de la Actividad Física y el Deporte, 49 (13), 1-17.

13. Scior, K. (2011). Public awareness, attitudes and beliefs regarding intellectual disability: A systematic review. Research in Developmental Disabilities, 32(6), 2164-2182.doi:10.1016/j.ridd.2011.07.005 